\title{
Telemedicine for reproductive medicine: pandemic and beyond
}

\author{
Denis A. Vaughan ${ }^{1,2,3,4}$ (D) Sophia H. Yin ${ }^{2,3,4}$. Jaimin S. Shah ${ }^{1,2,3,4}$. Annika Gompers ${ }^{2,3,4}$. Michele R. Hacker ${ }^{2,3,4}$. \\ Denny Sakkas ${ }^{1} \cdot$ Alice Domar $^{1,2,3,4} \cdot$ Thomas L. Toth $^{1,2,3,4}$
}

Received: 19 October 2021 / Accepted: 17 December 2021 / Published online: 13 January 2022

(c) The Author(s), under exclusive licence to Springer Science+Business Media, LLC, part of Springer Nature 2022

Utilization of telemedicine during the COVID-19 pandemic has allowed increased access to care while limiting in-person visits and risks of viral transmission [1]. The American Society for Reproductive Medicine and European Society of Human Reproduction and Embryology recommended limiting in-person consults and instead using telemedicine at the start of the pandemic, and telemedicine continues to be widely used even as guidelines have allowed for more inperson appointments at infertility centers $[2,3]$. Prior to the pandemic, telemedicine options were found to reduce barriers to healthcare access, particularly for patients who live farther away from clinics, with high patient satisfaction and, importantly, without affecting treatment outcomes $[4,5]$. There are also economic benefits for patients of less travel time and time off work. However, prior to the pandemic, telemedicine consultations were reimbursed at lower rates than in-office visits, rendering it challenging for healthcare organizations to provide it as a viable alternative. In midMarch 2020, the US Medicare program expanded coverage to telephone and videoconference visits for all patients, a change that was largely followed by other insurers [6]. Given this recent paradigm shift as a result of COVID-19, we sought to ascertain patients' perceptions of telemedicine modalities and their preferences for future infertility consultations.

Denis A. Vaughan and Sophia H. Yin contributed equally to this work.

Denis A. Vaughan

DVaughan@bidmc.harvard.edu

1 Boston IVF - The Eugin Group, 130 2nd Ave, Waltham, MA 02451, USA

2 Department of Obstetrics and Gynecology, Beth Israel Deaconess Medical Center, Boston, MA, USA

3 Department of Obstetrics, Gynecology and Reproductive Biology, Harvard Medical School, Boston, MA, USA

4 Harvard Medical School, Boston, MA, USA
All patients who attended a large, university-affiliated infertility practice in New England, USA, between January 1, 2019, and April 1, 2020, were included. The initial questionnaire was distributed to eligible patients from April 9 to 16,2020 . The study was determined to be exempt from review by the Institutional Review Board at Beth Israel Deaconess Medical Center (2020P000322). We received 3604 complete responses (response rate 34\%) [7] via RED$\mathrm{Cap}^{\mathrm{TM}}$ [8], a secure, HIPAA-compliant web application for online surveys. Using this platform, we linked consecutive questionnaires from the same individual, while maintaining anonymity. The same cohort of patients who initially responded received another questionnaire on January 1, 2021, with 1855 complete responses (51\% response rate). Analysis was restricted to patients who were not pregnant and actively pursuing infertility treatment $(n=1119)$. We used chi-square or Fisher's exact test to compare categori$c$ cal variables. Paired data was compared using a paired $t$-test for continuous variables and McNemar's test for categorical variables. For all tests, $p<0.05$ was considered statistically significant. All analyses were performed in SAS 9.4 (SAS Institute, Cary, North Carolina).

The majority of respondents self-identified as non-Hispanic White were married or in a domestic partnership, had completed college, and were employed. In April 2020, just before the peak of the first pandemic surge in the New England area, the majority of respondents selected "in person" as their preferred consult modality ( $58.1 \%)$, but by January 2021 , the top preference shifted to "video telemedicine" $(53.4 \%, p<0.001)$ (Table 1). Overall, in January 2021, respondents' top preference for modality of future consults was to have a combination of telemedicine and in-person consults (combined modalities with majority via telemedicine video/telephone $(42.3 \%)$ or combined modalities with majority in person (32.1\%)).

A secondary analysis demonstrated that age, race/ethnicity, completion of college, and household income were factors significantly associated with patient preference for consult modality (all $p<0.05$ ). Non-Hispanic White respondents 
Table 1 Respondents' perspectives on future appointment method (respondents who completed surveys in April 2020 and January 2021, $n=1119$ )

\begin{tabular}{|c|c|c|c|}
\hline & $\begin{array}{l}\text { April } 2020 \\
n(\%)\end{array}$ & $\begin{array}{l}\text { January } 2021 \\
n(\%)\end{array}$ & $p$ \\
\hline Should infertility treatments be offered during the COVID-19 pandemic? & & & $<0.0001$ \\
\hline Yes & $337(30.1)$ & $1040(92.9)$ & \\
\hline No & $61(5.5)$ & $6(0.5)$ & \\
\hline Unsure/depends/missing & $721(64.4)$ & $73(6.5)$ & \\
\hline Top preference for consult modality & & & $<0.0001$ \\
\hline In-person & $650(58.1)$ & $388(34.7)$ & \\
\hline Telephone & $201(18.0)$ & $128(11.4)$ & \\
\hline Telemedicine (video) & $261(23.3)$ & $598(53.4)$ & \\
\hline Missing & $7(0.6)$ & $5(0.5)$ & \\
\hline Preferred consult modality after the COVID-19 pandemic & & & $<0.0001$ \\
\hline All in-person consults & $219(19.6)$ & $200(17.9)$ & \\
\hline Majority of consults in person with occasional telemedicine video/telephone & $469(41.9)$ & $359(32.1)$ & \\
\hline Majority of consults via telemedicine video/telephone with occasional in person & $346(30.9)$ & $473(42.3)$ & \\
\hline All consults via telemedicine video/telephone & $69(6.2)$ & $67(6.0)$ & \\
\hline Missing & $16(1.4)$ & $20(1.8)$ & \\
\hline
\end{tabular}

Data presented as median with interquartile range or $n$ (column \%)

(54.4\%), non-Hispanic Black respondents (50.0\%), and particularly non-Hispanic Asian respondents (70.4\%) selected video telemedicine as their top consult modality. In contrast, Hispanic respondents (59.5\%) and those who self-identified as another race/ethnicity (49.1\%) were more likely to prefer in-person consults as their top consult modality $(p<0.001)$.

Higher household income also was associated with a greater preference for video telemedicine $(p<0.001)$, ranging from $36.7 \%$ for respondents whose annual household income was $<\$ 50,000$ to $62.0 \%$ for respondents with an annual household income $\geq \$ 200,000$. Full-time employment $(54.5 \%$ chose telemedicine compared to $50.5 \%$ of those without full-time employment) and having children (58.5\% chose telemedicine compared to $52.0 \%$ of those without children) were not significantly associated with modality preference ( $p=0.09, p=0.11$ respectively). Satisfaction with video telemedicine was very high (median score of 5 out of 5, IQR 4-5).

Importantly, having a previous telemedicine consult with a reproductive endocrinologist and infertility (REI) physician was associated with a higher preference for telemedicine in the future, signaling greater comfort with this modality with prior experience. At our center, we began using telemedicine services for consultations in March 2020. In April 2020, only $10.5 \%$ of respondents previously had an appointment via video telemedicine, but by January 2021, $69.1 \%$ of respondents had. Among patients with a previous telemedicine consult, $62.5 \%$ chose video telemedicine as their top consult modality, $6.9 \%$ chose telephone, and $30.7 \%$ chose in person. By comparison, of those who did not have a previous telemedicine experience with a REI physician, $33.7 \%$ preferred video telemedicine, $22.0 \%$ preferred telephone, and $44.3 \%$ preferred in-person consults $(p<0.001)$. Also, a greater proportion of respondents with a previous telemedicine experience preferred their future consults to be mostly via video telemedicine with occasional in-person visits (45.5\%) or all via video telemedicine (6.6\%) compared to those who did not have a previous telemedicine consult (37.6\% and 5.0\% respectively, $p<0.001$ ).

Previous examinations of the impact of telemedicine have demonstrated that it can be used in infertility consults with high efficacy and patient satisfaction $[4,5]$ Our study further explored patient preferences, and importantly, examined patient preferences in the presence of a pandemic. We found that patients value flexibility of consult modality and prefer to continue having access to telemedicine options for infertility consults even beyond the pandemic. Given the association between a higher preference for telemedicine with prior experience seen in this study, we expect favorable views of this modality to continue to increase with increased use.

Limitations of this study include its response rate, which may result in a sample that is not representative of all patients pursuing infertility treatment. In addition, the survey was completed in the setting of a pandemic, and views about telemedicine outside of a pandemic may differ. The sample was limited to the New England area of the USA and may not be generalizable to other regions.

Telemedicine provides a highly satisfactory alternative modality for infertility consults, which are largely discussion-based and do not always involve a physical 
examination of the couple. In the midst of a pandemic, it allowed patients to safely access care while mitigating the stressor of COVID-19 [7] As we look to the future, the large majority of respondents (74.4\%) favor a combination of inperson and telemedicine consults. Patient preference varies by patient self-identified race/ethnicity, a relationship that has been also noted in telemedicine use in primary care [9]. Patients with lower household incomes tended to prefer inperson visits as compared to those with higher household incomes, which may reflect differential access to technology or other barriers. Further studies should examine the reasons for these noted differences. The benefits of telemedicine for patients, such as less time off work and fewer expenses for childcare and transportation, as well as the differences between the trend towards an increased preference for video telemedicine rather than telephone consults, may be the subject of future examinations.

As policymakers and commercial insurance carriers examine coverage moving forward, the option to have consults via telemedicine modalities promotes patient satisfaction, provides economic benefits to the patient, and perhaps, most importantly, allows increased access to care. Telemedicine may allow healthcare provision to patients who may be unable to physically present to a clinic due to geographic, time, or employment-related barriers, as well as increase patient convenience. Addressing these barriers may help decrease inequities in access and care. The choice of consult modality should rest as a joint decision-making process between patient and practitioner.

Funding This study was supported by a COVID-19 Investigational Grant in Reproductive Medicine and Maternal Health (RMMH), sponsored by Ferring Pharmaceuticals and Harvard Catalyst I The Harvard Clinical and Translational Science Center (National Center for Advancing Translational Sciences, National Institutes of Health Award UL1 TR002541) and financial contributions from Harvard University and its affiliated academic healthcare centers.

Data availability Data available on request from the corresponding author, DAV.

Code availability Not applicable.

\section{Declarations}

Ethics approval The study was determined to be exempt from review by the Institutional Review Board at Beth Israel Deaconess Medical Center on 7 April 2020 (IRB protocol number: 2020P000322).
Consent to participate Patients consented to participate in this study by completing the questionnaires.

Consent for publication N/A.

Conflict of interest The authors declare no competing interests.

\section{References}

1. Weiner JP, Bandeian S, Hatef E, Lans D, Liu A, Lemke KW. Inperson and telehealth ambulatory contacts and costs in a large US insured cohort before and during the COVID-19 pandemic. JAMA Netw Open. 2021;4(3):e212618-e212618.

2. Medicine ASfR. Patient management and clinical recommendations during the coronavirus (COVID-19) pandemic. https:// www.asrm.org/news-and-publications/covid-19/statements/patie nt-management-and-clinical-recommendations-during-the-coron avirus-covid-19-pandemic/ March 17, 2020.

3. Embryology ESoHRa. Assisted reproduction and COVID-19: a statement from ESHRE for phase 1 - Guidance on fertility services during pandemic. https://www.eshre.eu/Europe/Positionstatements/COVID19 April 2, 2020.

4. Mikhael S, Gaidis A, Gavrilova-Jordan L. Regional disparities in access to assisted reproductive technology: assessment of patient satisfaction when employing modern technology to close the gap. J Assist Reprod Genet. 2021;38(4):889-94.

5. Alexander VM, Schelble AP, Omurtag KR. Traits of patients seen via telemedicine versus in person for new-patient visits in a fertility practice. F S Rep. 2021;2(2):224-9.

6. President Trump Expands Telehealth Benefits for Medicare Beneficiaries During COVID-19 Outbreak [press release]. U.S. Centers for Medicare \& Medicaid Services, March 17, 2020.

7. Vaughan DA, Shah JS, Penzias AS, Domar AD, Toth TL. Infertility remains a top stressor despite the COVID-19 pandemic. Reprod Biomed Online. 2020;41(3):425-7.

8. Harris PA, Taylor R, Thielke R, Payne J, Gonzalez N, Conde JG. Research electronic data capture (REDCap)-a metadatadriven methodology and workflow process for providing translational research informatics support. J Biomed Inform. 2009;42(2):377-81.

9. Reed ME, Huang J, Graetz I, et al. Patient characteristics associated with choosing a telemedicine visit vs office visit with the same primary care clinicians. JAMA Netw Open. 2020;3(6):e205873. https://doi.org/10.1001/jamanetworkopen. 2020.5873 .

Publisher's note Springer Nature remains neutral with regard to jurisdictional claims in published maps and institutional affiliations. 